\title{
Spiritual Care for Critical Care Patients
}

\section{Alham Abuatiq}

College of Natural Sciences, Department of Nursing, California State University, San Bernardino, CA 92407-2318, USA

\begin{abstract}
Aims: The purpose of this manuscript is to present a theoretical perspective of the spiritual care in the critical care setting, and present the clinical art of implementing the spiritual care for critically ill patients. Background: A theoretical frame work is crucial in building a therapeutic spiritual care for critically ill patients. Neuman's nursing theory (1970) is presented as an example to the early recognition of spiritual care in the nursing science. A more modern view of the spiritual care structured in the synergy model which is presented by the American Critical care association in (1997). A case report is presented as an operational implementation of spiritual care in critical care units.

Implications: Spiritual care is a patient centered care, implementing an individualized spiritual care is influenced by the critical care environment, presence of a spiritual oriented patient, family members and health care providers. Precise assessment of patients' needs will enhance the conduction of effective spiritual care. Simple interventions like being with the patient, listening to them, emphasizing on the importance of balancing spirit, mind and body needs; and making appropriate referrals may help patients in their spiritual healing process.

Significance of spiritual care in critical care: Patient oriented spiritual care plan is crucial to provide effective and efficient recovery from critical illness. Patients who have their spiritual needs addressed and received spiritual care, are less anxious, have less stress, enhanced coping, and have better prognosis from illness.
\end{abstract}

\section{Introduction}

The purpose of this manuscript is to present a theoretical base of the spiritual care in the critical care setting, and present the clinical art of implementing a spiritual care for critically ill patients. A theoretical frame work is important in building a therapeutic spiritual care for critically ill patients. Neuman's nursing theory (1970) is presented as an example to the early recognition of spiritual care in the nursing science. A more modern view of the spiritual care structured in the synergy model which is presented by the American Critical care association in (1997)

\section{Theoretical Framework}

Many theoretical models integrated the spiritual dimension of patients care, for example the neuman systems model which was originally developed in 1970 provides a conceptual frame work and representation of humans and nurses interactions in the health care setting.

Neuman's model represented the person is an open system and is viewed a "layered multi dimensional being, each layer consists of five subsystems that include: the physical dimension refers to the physicochemical structure. The Psychological reflected by the mental process and emotions. The sociocultural refers to the social relationships and sociocultural expectations. The developmental: refers to process related to development over the life span. And spiritual dimension that refers to the influence of spiritual beliefs [1].

Persons are surrounded by the Environment in which they interact at any given time. Persons are in optimal health and wellness condition in which all layers of the open system are in harmony with the whole of the person, as the person is in a constant interaction with the environment; the state of wellness is in a dynamic equilibrium to achieve the optimal harmony [1]. Nursing care is concerned with all the dimensions mentioned and influences the responses the person may have toward a stressor.

The goal of neuman model is to achieve optimal system (patient) stability and satisfaction to overcome both internal and external stressors that the patients may suffer from.

Neuman systems model is an excellent model for nurses and other health care professionals to think about stress and stress prevention, however, one of the criticisms include : "While it is useful to think of people as layered and made up of five principles, it is not easy to predict or describe their interplay. Moreover; since each layer is composed of all the person variables, it is not always clear as to what layer is being assessed in any operationalized variable" [1].

In the critical care units the focus is more on stabilizing the patients' physiological status. High priority is given to maintain adequate oxygenation, perfusion and nutrition to the critically ill patient's body. Nurses have to deal with many sedative, vasoactive, and other medications or procedures. Nursing care is directed to control and stabilize the patients physiologically more than focusing on their spiritual needs. With the progress of time, the ICU environment became more complex as well as the complexity of care and medical interventions provided for critically ill patients.

The American association of critical care nurses (AACN) identified that establishing a healing environment is considered as one of the research priorities. The standards for establishing a healthy work environment include providing a safe, healing, and respectful of the rights, responsibilities, needs and contributions of patients, patient's families, and all health care providers [2].

AACN synergy model is a standard conceptual framework for critical care nursing, the patient is cared for as a whole person: mind,

"Corresponding Author: Dr. Alham Abuatiq, College of Natural Sciences, Department of Nursing, California State University, San Bernardino, CA 924072318, USA; E-mail: aabuatiq@csusb.edu

Citation: Abuatiq A (2015) Spiritual Care for Critical Care Patients. Int J Nurs Clin Pract 2: 128. doi: http://dx.doi.org/10.15344/2394-4978/2015/128

Copyright: () 2015 Abuatiq. This is an open-access article distributed under the terms of the Creative Commons Attribution License, which permits unrestricted use, distribution, and reproduction in any medium, provided the original author and source are credited. 
body and spirit [3], the nursing care should be provided in to match with the patient physiological, psychological, and spiritual needs. The synergy model identifies many nurses and patients characteristics interacting in the health care setting. Figure-1 represents the Synergy Model [4].

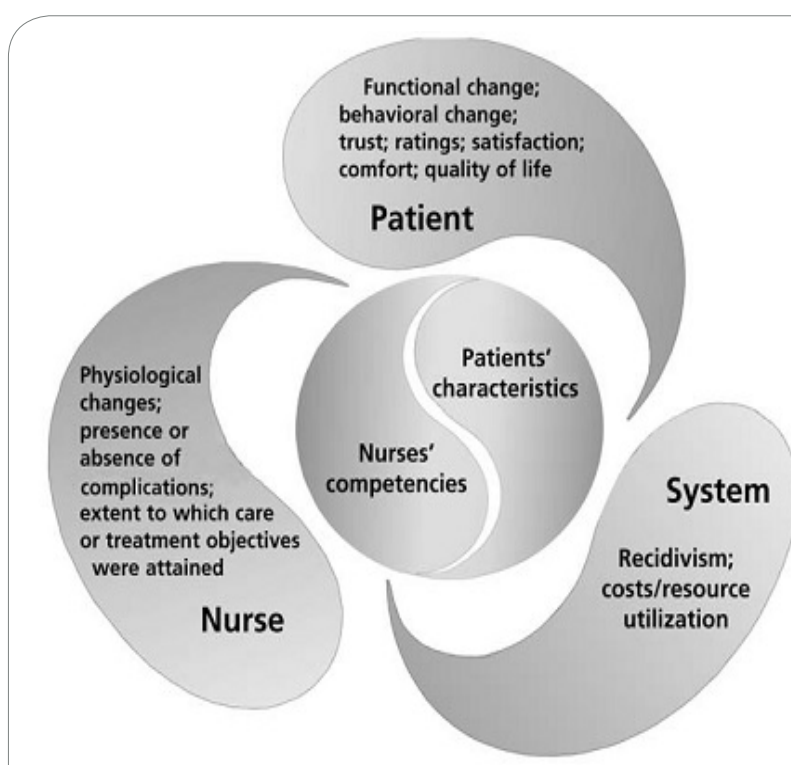

Figure 1: The American Association of Critical Care Nurses Synergy Model for Patients Care (Curley, M. 1998).

\section{Assumptions of the AACN Synergy Model}

The assumptions of the synergy model are: "Patients are biological, psychological, social and spiritual entities who present at a particular developmental stage. The whole patient (body, mind and spirit) must be considered. The patient, family and community all contribute to providing a context for the nurse-patient relationship. Patients can be described by a number of characteristics; all are connected and contribute to each other. Similarly, nurses can be described on a number of dimensions. The interrelated dimensions paint a profile of the nurse. A goal of nursing is to restore a patient to an optimal level of wellness as defined by the patient. Death can be an acceptable outcome, in which the goal of nursing care is to move a patient toward a peaceful death" [2].

According to this model nursing is based on establishing the most effective relationship between a nurse and a patient according to the patient needs. Matching the nurse's competencies according to the patient needs and required care. And the most important aspect is the spiritual care. Nurses should establish two characteristics according to this model which include: caring practices and response to diversity. On the other hand, patients' characteristics include resilience and resource availability. To operationally apply this model to care for a critically ill patient with spiritual needs case report is presented below:

\section{Case Report}

It was shortly after the night shift report; an ICU registered nurse was assigned to two critical care patients. One of the patients Mrs. B. is admitted to the intensive care unit due to neutorpnia status and septic shock after receiving a course of chemotherapy. the nurse entered the patients room to do the initial assessment, the patient was Conscious oriented to person and place did not remember the time, she was anxious, heart rate above 110 beat/ $\mathrm{min}$. when the nurse asked her how was your day, the patient replied: "the doctor came to me in the morning and told me that my cancer is spreading fast and the treatment may not be effective. I have one daughter and I do not want to die, she will not have any one to take care of her, I took the chemotherapy and I do not think that any medication or surgery will treat my cancer, I am really afraid to die, it is not my time yet, I have requested a chaplain to visit me but nobody came and now it is night time, what should I do, I did not sleep since 2 nights thinking about my destiny?". The nurse replied: "I will request the chaplain again to visit you?". The patient replied: "God is punishing me, I spent all my life working in a bar and rarely went to church, I even do not memorize any prayers". The nurse answered: "do you need any specific prayers book to help you?" The patient answered "no, reading prayer books is boring; I prefer to listen to prayers or join another person in praying". The nurse answered : "We can open the TV and you can watch the prayers if you are interested in?" the patient answered:" I hope this will work, let us search for a channel" the patient selected a catholic prayers channel and started watching it. The nurse stayed for a minute with her, checked the patient's monitor connections. The nurse was worried about the other patient whose cardiac monitor was alarming for low blood pressure. The nurse went to the other patient to stabilize his condition. After 30 minutes the daughter came to visit her mother and joined her mother in watching the prayers. The priory in the ICU setting is to control the physiological parameters of the patients. Mrs. B heart rate became more stable during the shift, slept more hours, she did not request any pain medications, she seemed less anxious and with comfortable facial expressions.

\section{Applying the synergy model in this case}

\section{Patient's resiliency and resource availability}

Resiliency is defined as "the capacity to return to a restorative level of function using compensatory coping mechanisms" [3]. The patient is considered to have minimal resiliency if they failed to compensate to any threat or injury. On the other hand, the highest level of resilience is achieved when the patient is coping effectively to the experienced threat.

The resource availability in patients depends on "the extent of resources brought to the situation by the patient, family and community" [3]. Resources include all personal, psychological, social, physical and financial resources that can be provided during the experience of threat or injury to the human body, mind and soul.

\section{Operational application}

According to the case study presented, the patient verbally expressed her resiliency accordingly:

1. From the assessment phase: injury or threat is expressed verbally by the patient, she is experiencing this phase of illness in which she consider it as a punishment from god. The patient expressed her need for social and psychological resources. The patient expressed that she wants to restore her level of function to take care of her daughter.

2. The resources that the patient identified to have more resiliencies include; requesting a chaplain, engaging in prayer activities by watching the TV religious channel and joining her daughter in prayers. The patient chose the resources that she feels comfortable with she preferred joining live religious practices rather than reading from a prayer book. 
3. Evaluation: prayer is considered a coping mechanism, the patient resilience could not be measured in numbers, however, qualitatively the patient had comfortable non verbal facial expressions, and became less anxious, was able to sleep more, and her heart rate became more stable.

\section{Nurses caring practices and response to diversity}

The nursing care practice is organized by the nursing care plan that includes assessment, planning, diagnosis, interventions and evaluation. The nursing care plan is a patient centered care. The goal of the nursing care is to provide effective therapeutic outcomes, increase patient's comfort, and prevent suffering. Nurses assess their patients spiritual practices from many resources like for example: patient's verbal expression of his own spiritual practices, presence of photos, symbols in patient's bed side, and presence of a family member who express or practice spiritual care.

Responses to diversity include the nurse's ability to assess and implement the most accurate spiritual interventions according to the patient's own spiritual needs. Nurses are obligated to learn about the spiritual beliefs and practices of their patients in order to provide to satisfactory patients care and achieve optimal outcomes.

\section{Operational application}

According to the case study presented, the critical care nurse performed:

1. Assessment of her patients spiritual needs, she asked open ended question to encourage the patient to have self disclosure. In the assessment phase the nurse listened to her patient and explored her patient's spiritual concerns. To guide the healthcare professionals on how to precisely assess their patients spirituality, healthcare providers can utilize the "FACT" spiritual assessment tool, Recommend to be used for spiritual assessment in the healthcare setting [6]. "FACT" spiritual assessment tool provides a protocol to ask patients about their spirituality during therapeutic conversation. "FCAT" tool acronym is explained in details as follows: "F: stands for Faith", "A: for Active, availability, \& accessibility", "C: for Coping", and "T: for Treatment plan" [6]. Healthcare practitioners can precisely assess their patient's spirituality utilizing this assessment tool as follows:

a. "Faith" Assessment: Healthcare providers can assess this aspect by asking open ended questions related to faith, suggested questions include asking the patient "What is your faith or belief?" "Do you consider yourself spiritual or religious?" [6].

b. "Active" Spiritual practice: the healthcare practitioner's assess their patients active spiritual practices by asking open ended question, recommend questions include "Are you active in your faith community? Or Is support for your faith available to you?" [6].

c. "Coping": healthcare providers can ask the patients to verbalize how they cope with the medical illness. Suggested questions to assess this phase include asking the patient "How are you coping with your illness?" , "Do any of your religious beliefs or spiritual practices conflict with medical treatment?" [6].

d. "Treatment plan": if the patient is coping well, healthcare providers should support, encourage and re-assess later [6], however if the patient is nor effectively coping, the healthcare providers should first, provide spiritual counseling, prayer, and provide direct interventions according to the patients spiritual preferences. Second, encourage their patients to their spiritual concerns with their faith/spiritual leader. And Third, make referral to the hospital religious figure [6] .
2. Plan and intervention: the nurse was attentive to her patients needs, she followed to the referral to chaplain, and she encouraged the patient to seek spiritual resources present in the patient's room by offering a prayer text book and opening the TV on patient's preferable religious channel.

3. Evaluation: spiritual care is an ongoing process; continuous assessment, planning and interventions are required to progress in the care provided, at the evaluation phase the patient may express feelings of satisfaction, and comfort.

\section{Conclusion of case study application}

To support the patient in her resiliency and enhance her coping, the nurse provided time, space, made appropriate referrals, encouraged the engagement of family member in the spiritual practice. Effective outcomes were observed in this case, however sometimes critical care nurses focus more on the physiological monitoring of the critically ill patients and forget to implement the spiritual practices required by their patients. Time, staff shortage and unstable patient's condition affect the spiritual practice in the critical care units.

\section{Spirituality in Critical Care and My Own Clinical Experience}

From my own experience in the critical care units, I have noticed that patient's families play a major role in directing the spiritual care of their patient. Providing religious icons, holy books grasp the attention health care providers and we are becoming more aware of the importance of spiritual care to prevent stress and enhance coping. Patients or family members who requested a chaplain will be granted this spiritual care.

In end of life cases, some patients stay in the ICU after changing their case to do not resuscitate, the patient will be provided with comfort care, allowing the natural death process.

In the critical care unit I used to work in nurses will put a white rose in the nursing station in front of the dying patient's room to send a message to all health care providers about the presence of a patient with end of life care needs. This simple symbol increased the courtesy and respect of health care providers to patients and their families' feelings. Comfort measures are provided to the dying patient, space and time for spiritual practices is given and in general prayers are directed by the patient's family.

A spiritual or religious entity will be provided per patients or families request. On the other hand, critical care patients who are in coma status, and with no known history of their own spiritual or religious practices rarely get a spiritual care, I have seen few cases in which the $\mathrm{TV}$ or radio will be turned on by the nurse in a religious channel as a courtesy to the patient's spiritual needs if any is recognized.

\section{Significance of Spiritual Care in Critical Care}

The human being consist of the spirit, mind and body, we feed our mind and body contentiously on daily bases. The spiritual nourishment depends of the individual's own spiritual beliefs and practices. Spiritual dimension forms the umbrella that includes the religion within. A person could not have a religion but still may be considered a spiritual person. On the other hand, a religious person is considered a spiritual person. Spiritual practices are the food for the soul. The soul may suffer a spiritual distress if the spiritual needs are not served.

In the critical care units the patient's body and mind are restored and treated by medications, nutrition is given via feeding tubes or 
Page 4 of 4

or parenteral nutrition. Being in critical illness may cause spiritual distress; physiological healing and coping may be delayed if the patient is having spiritual distress. Patient oriented spiritual care plan is crucial to provide effective and efficient recovery from critical illness. Patients who have their spiritual needs addressed and received spiritual care, are less anxious, have less stress, enhanced coping, and have better prognosis from illness.

The joint commission on accreditation of health care Organizations (JCAHO)[7] requires the administration of spiritual assessment. The spiritual care in critical care is directed according to the patient's needs. spiritual distress is an accredited nursing diagnosis according to NANDA [8], nurses should implement interventions to resolve the spiritual distress their patients suffer from, This nursing care does not mean that nurses should provide a pure religious practice to the spiritually distressed patient, simple interventions like being with the patient, listening to them, emphasizing, and making appropriate referrals may help in their healing process. Moreover; nurses can encourage patients to practice their own spiritual beliefs to assist in convalescence and enhance coping.

\section{Implications of Spiritual Care in Critical Care Setting}

1. Spiritual care in the critical care environment: The critical care environment is filled with monitors, alarms, ventilators, cardiac monitors and other life support machines; this environment is a challenge when it comes to providing spiritual care. Space and time and spiritual entity are required to implement an effective spiritual care. Many health care providers prioritize their interventions to control their patient's physiological status. We have to always remember that integrating and prioritizing the spiritual care have equal effectiveness for improving the patient's condition or easing their death process.

2. Spiritual care and critical care patients: The majority of critical care patients are sedated, intubated and connected to life support machines, the patients may not be able to perform spiritual activities during this phase. Many patients who recovered from those conditions remember conversations that occurred in their bedside. Listening is the most active sense. We can utilize this sense to provide the spiritual needs of the patient. For example: turning on slow music, turning the TV on the patient's preferable religious channel if any. Spiritual care is patient centered, holistic assessment of patient spiritual and religious history may guide the providers to their patient's specific spiritual activities if any is present.

3. Spiritual care and critical care patient's families: In general, patient's families can help the patient in the spiritual care; they provide religious icons, books and pray for and with the patient. The presence of family near the patient is crucial for their spiritual care.

4. Spiritual care and Health care providers: we as health care providers have to be aware about our own spiritual health; we cannot provide something we do not have, so self realization, education and spiritual fulfillment is required from health care providers. Different spiritual believes and an expectation of patient's forms a significant challenge to health care providers. The spiritual care is highly personal and individual care based. We as health care providers have to be prepared with the knowledge and skills to respond effectively to the diversity of cultural and religious practices and implement the care in a patient centered framework.

\section{Summary}

This paper presented a theoretical perspective of spiritual care. Case report is presented to reflect an example of spiritual practices present in the critical care setting. The significance of spiritual care is essential to patient's whole integrity. The current health care is patient centered and patient outcomes focused. Specialized religious or spiritual members in the health care team are more involved in the patients care. The patients centered care practice that we implement in the health care setting could make the patients spiritual care more accessible and doable.

\section{Competing Interests}

The author declare that she has no competing interests.

\section{References}

1. Neuman's Model (2013) Nursing theory website.

2. Wolfe HP, Neuman S (2000) System Model Criticism.

3. The American Association of Critical Care Nurses Synergy Model Assumptions (2014).

4. Hardin S, Kaplow R (2005) Synergy for Clinical Excellence: the American Association of Critical Care Nurses Model for Patients Care. Boston, Mass: Jones \& Bartlett publishers Inc.

5. Curley M (1998) Patient nurse synergy: optimizing patients' outcomes. Am J Crit Care 7: 69-71.

6. LaRocca-Pitts M (2009) In FACT, Chaplains have a spiritual Assessment Tool. Australian Journal of Pastoral Care and Health 3: 8-15.

7. The Joint Commission Accreditation Standards (2014).

8. NANDA International (2014). 